\title{
Blue Light Imaging and Linked Color Imaging for the Characterization of Mucosal Changes in Chronic Gastritis: A Clinicians View and Brief Technical Report
}

\author{
Jochen Weigt $^{\mathrm{a}} \quad$ Peter Malfertheiner $^{\mathrm{a}} \quad$ Ali Canbay $^{\mathrm{a}} \quad$ Johannes Haybaeck $^{\mathrm{b}}$ \\ Elizabeth Bird-Lieberman ${ }^{c}$ Alexander Link ${ }^{a}$

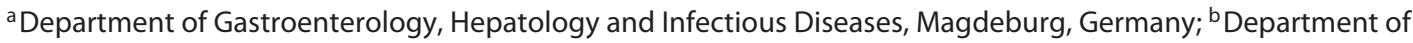 \\ Pathology, Otto von Guericke University Magdeburg, Magdeburg, Germany; ${ }^{\mathrm{C}}$ Translational Gastroenterology Unit \\ and NIHR Oxford Biomedical Research Centre, Oxford University Hospitals NHS Foundation Trust, John Radcliffe \\ Hospital, Oxford, UK
}

\section{Keywords}

Blue light imaging · Linked color imaging · Chronic gastritis · Atrophic gastritis · Intestinal metaplasia

\begin{abstract}
Background: Blue light imaging (BLI) and linked color imaging $(\mathrm{LCl})$ are new imaging modalities for the endoscopic evaluation of mucosal changes within the digestive tract. There is little experience with these modalities in the characterization of chronic gastritis (CG) intestinal metaplasia (IM) and atrophy in the stomach. Aims and Methods: In a singlecenter observational pilot study, we correlated endoscopic findings with histology in selected patients. Results: Findings from 29 patients were included in the analysis. Six patients had macroscopically normal gastric mucosa at endoscopy, and this was confirmed histologically in 5 of them. At endoscopy, 15 patients had the presence of IM in the antrum predicted, and this was confirmed histologically in 11 (73\%). In the corpus, we predicted the presence of IM in 14 patients, and this was confirmed in 11 (78\%) at histology. Eleven patients had the endoscopic suspicion of atrophy in antrum, which was confirmed in 9 patients (82\%). In total, 14 patients
\end{abstract}

(c) 2019 S. Karger AG, Basel

\section{E-Mail karger@karger.com www.karger.com/ddi \\ KARGER}

had endoscopic suspicion of atrophy in corpus mucosa at endoscopy, but only 10 were confirmed in histology (71\%). The concordance of endoscopic classification and histology was $93 \%$ for antrum and $88 \%$ for corpus. The positive predictive value and negative predictive value for IM were 0.74 and 0.83 and for atrophy 0.63 and 0.97 , respectively. Conclusions: $\mathrm{LCl}$ and $\mathrm{BLI}$ are helpful in characterization of mucosal changes in CG. The ability to rule out premalignant conditions by endoscopy only reflects the clinical use and harbors significant clinical implications.

(c) 2019 S. Karger AG, Basel

\section{Introduction}

The current and widely accepted gold standard for the endoscopic characterization of mucosal changes in the upper gastrointestinal (GI) tract is the use of virtual chromoendoscopy, with magnification that enables visualization of the pit pattern and vascular changes [1-3]. Narrow band imaging (NBI) is at present the best characterized method. It works with an optical filter that allows only small bands of light to pass, which results in enhancement

PD Dr. med. Jochen Weigt

Department Gastroenterology, Hepatology and Infectious Diseases

Otto-Von Guericke University Magdeburg

Leipziger Strasse 44, DE-39120 Magdeburg (Germany)

E-Mail jochen.weigt@ @med.ovgu.de 
of the mucosal vasculature and pit pattern. NBI use for the characterization of neoplastic or inflammatory changes in the GI tract has been investigated intensively.

Recently, a new method using an equivalent physical principle was developed. Here a laser of $410 \mathrm{~nm}$ is used to create a highly narrowed blue band together with other wavelengths that are continuous in spectrum but with lower intensity $[4,5]$. Image results show similarities to NBI; however, NBI filters the visible spectrum of a Xenon lamp, thus resulting in a light of lower intensity. In contrast, blue laser light is directly transmitted without a filter, resulting in higher light intensity.

The most recent development in light-emitting technology is the so-called multilight illumination (MLI) that composes light from 4 different light emitting diodes (LED) [6]. By intensifying the amount of light, emitted by each LED with a different intensity, a set of specific light modes can be achieved.

Blue light imaging (BLI) is characterized by a continuous light spectrum with intensity peaks at 410 and $450 \mathrm{~nm}$, enhancing surface and vascular structures. However, owing to its continuous light spectrum, it also enhances the visibility of other structures, such as vessels more deeply located in the submucosa. In addition, the white light mode of MLI does not show a continuous light spectrum that is comparable to xenon white light, which is characterized by a homogenous intensity of all wave lengths, but is characterized by peaks in a continuous spectrum.

Linked color imaging (LCI), a mode composed of bright BLI light combined with a postprocessing algorithm, which shows colors that are more similar to a white light image but with an increased red-to-red contrast. This is achieved by reallocation of colors in the color space [7, 8]. Reallocation means that the color wavelengths that are detected by the CMOS sensor are replaced by another color wavelength on the display. This leads to an increase of the amount of displayed colors and therefore automatically to an increase in color difference.

Despite the high likelihood that this technique would deliver images similar to NBI, robust data are needed to investigate this new technology, as some remarkable differences to other virtual chromoendoscopy technologies exist. The greater light strength delivered and spectral composition has the potential to provide additional data, which could improve imaging compared to NBI. At present, data on the value of characterization mucosal changes with BLI are scarce, irrespective of whether they are used with the laser system, available on Asian markets only, or with MLI, available only in Europe.
There is great clinical interest in the ability to detect chronic gastritis (CG), intestinal metaplasia (IM), and atrophy at endoscopy since this has the potential to minimize sampling error, decrease unnecessary biopsies, and allow assessment of the distribution of these lesions. The current MAPS guidelines concerning premalignant conditions in the stomach recommend the follow-up of patients with atrophy or IM only when extensive disease is present [9]. However, the exact distribution of histologic changes can only be defined by improved endoscopic identification of these conditions and by careful and well documentation of where samples have been taken. For instance, a histology report of a patient with only a focal area of IM both in the antrum and corpus can lead to 3 different interpretations of reports. If biopsies were only taken from the lesion or area of concern, the histological report could be falsely interpreted as a general distribution of IM in antrum and corpus. If only tissue outside the lesion was sampled, the stomach would be classified as being completely normal, and if both the lesion and adjacent tissue were sampled, still information about the distribution of mucosal changes would not be available. Therefore, only histology together with endoscopic reporting can help to stage CG correctly.

In this study, we investigated the predictive value of BLI with MLI for the characterization of gastric mucosal changes in a small cohort using histology as the gold standard. In addition, we give technical insight in this new imaging modality.

\section{Methods}

This study is a single-center uncontrolled analysis of prospectively selected patients with clinical suspicion to have CG or with already diagnosed CG in advanced stages, for example, mucosal atrophy or IM. Patients were included over a period of 12 weeks.

The study adheres to the declaration of Helsinki and data protection law.

All patients gave their written informed consent prior to endoscopy. They were sedated with propofol, and endoscopy was performed in a left lateral position.

All patients were investigated using the EG 760ZP gastroscope (Fujifilm, Japan). The light source and a video processor was Eluxeo (Fujifilm, Japan) with 30-fold magnification. Images of all anatomic sides and focal lesions were taken with all different available light modes. Endoscopy was always carried out in the following sequence. First white light intubation of duodenum. Withdrawal into the stomach. Inspection using WLE followed by LCI including antegrade and retrograde positions. Focal lesions were inspected using BLI afterward.

Endoscopic classification was based on the following parameters:
10

Dig Dis 2020;38:9-14

DOI: $10.1159 / 000501265$
Weigt/Malfertheiner/Canbay/Haybaeck/ Bird-Lieberman/Link 

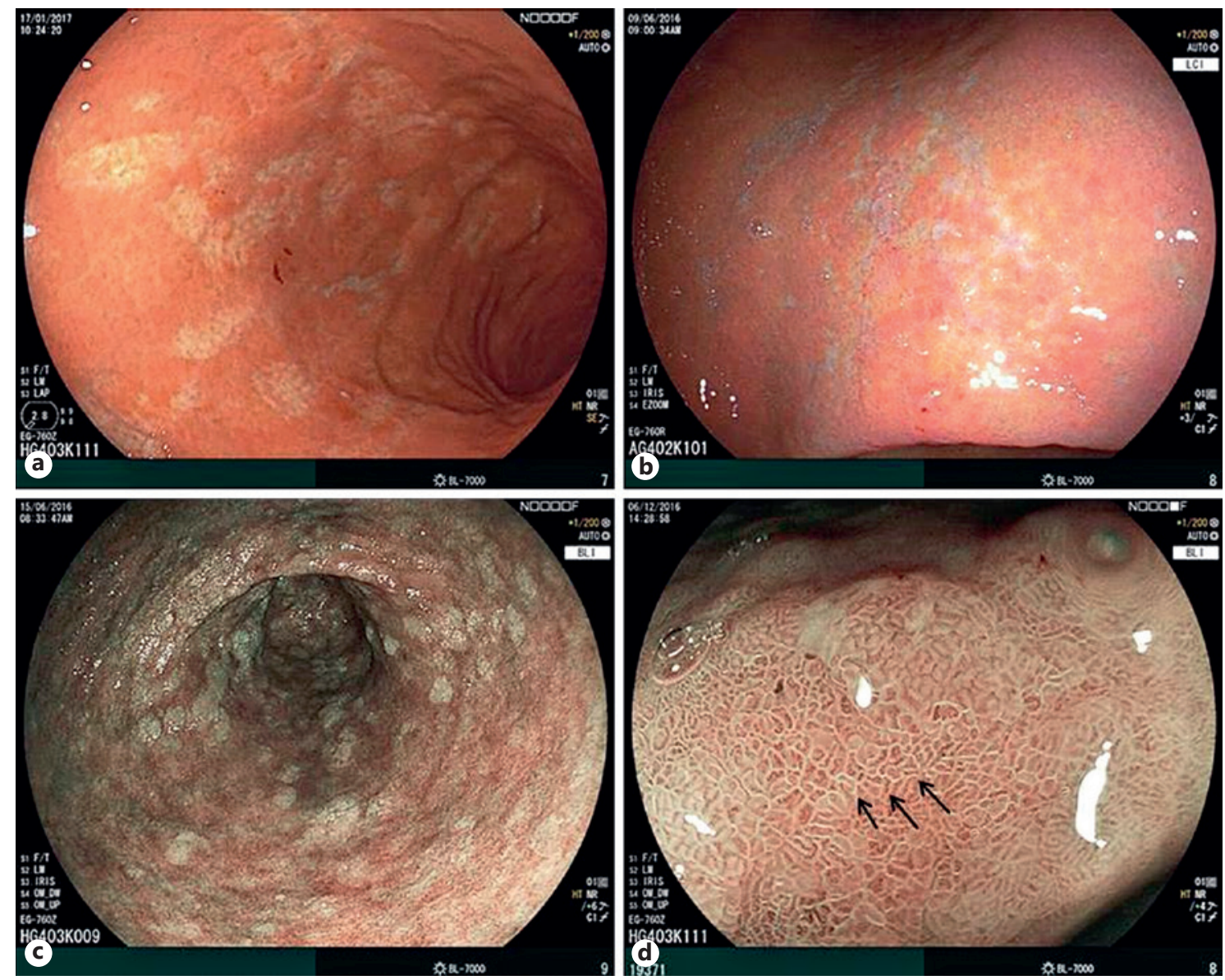

Fig. 1. Typical images for gastric pathology using MLI. a, b Same patient, (c, d) different patients. a Overview image of CG with larger areas of mucosal atrophy with a yellow appearance in white light. b Mucosal atrophy at the lesser curvature using LCI atrophy

a. Normal gastric mucosa was defined as mucosa with visible superficial capillary network without any focal lesions.

b. Atrophy gastritis was defined by whitening of the mucosa with visible deeper vascular architecture in white light, BLI and LCI or the visibility of deeper tortuous pits.

c. IM of the mucosa was defined by villous or ridged surface with whitening in LCI or white light or light blue crest sign in BLI.

d. CG was diagnosed in case of loss of superficial capillary network or focal lesions not matching the definition of other focal lesions or cancer.

Biopsies were taken from every identified focal lesion additional to the updated Sidney classification system (2 samples antrum, 2 samples corpus). Helicobacter pylori testing was routinely performed in all patients using rapid urease test and histology. Guidance of targeted biopsies was performed under BLI with or without magnification with the open forceps in the nearest position to the working channel. The biopsy forceps were closed after reaching the targeted lesion to assure correct sampling. During the examination, the endoscopist predicted the histology and the distribution of changes separately for antrum and corpus in the report. See Figure 1 for examples of the endoscopic findings. The results of appears white and deeper vascular structures can be visualized. c Patchy distribution of IM in the antrum appearing as white areas in BLI mode. $\mathbf{d}$ Magnification of angulus revealing light blue crest sign (arrows) as a sign of IM.

histology and endoscopy were correlated. Therefore, 3 clinically significant outcomes were defined: 1 st exact match between endoscopy prediction and histology, 2nd histology and endoscopy produce different results but still lead to the same recommendation as regards the follow-up, and 3rd histology and endoscopy do not correlate, and endoscopy alone may have led to an incorrect follow-up.

\section{Results}

In total, 29 patients were included in the analysis (15 females, 14 males, age 65 years [25-85 years]). Six patients were predicted to have normal gastric mucosa on endoscopy, which was confirmed histologically in 5 patients. The other patient had mild CG on histology. Endoscopically 15 patients had IM predicted in the antrum. Of these 15 patients, $11(73 \%)$ were confirmed on histology. In the 
Fig. 2. Graphical summary of the results showing the comparison of endoscopic and histologic findings in all patients. The diameter of the connection paths expresses the number of cases. Note that there are more connections changing the final diagnosis in the antrum compared with the corpus demonstrating a higher level of uncertainty. CG, chronic gastritis; IM, intestinal metaplasia; AG, atrophy gastritis.

\begin{tabular}{|c|c|c|}
\hline \multirow{7}{*}{ Corpus } & $\begin{array}{l}\text { Endoscopic } \\
\text { diagnosis }\end{array}$ & Histology \\
\hline & Normal & Normal \\
\hline & CG & CG \\
\hline & IM & IM \\
\hline & AG & AG \\
\hline & $\mathrm{IM}+\mathrm{AG}$ & $I M+A G$ \\
\hline & Cancer & Cancer \\
\hline \multirow[t]{7}{*}{ Antrum } & $\begin{array}{l}\text { Endoscopic } \\
\text { diagnosis }\end{array}$ & Histology \\
\hline & Normal & Normal \\
\hline & CG & CG \\
\hline & IM & $\mathrm{IM}$ \\
\hline & AG & AG \\
\hline & $I M+A G$ & $I M+A G$ \\
\hline & Cancer & Cancer \\
\hline
\end{tabular}

corpus, we found 14 patients predicted to have IM an endoscopy and 11 (78\%) confirmed on histology. Eleven patients had suspicion of atrophy in the antrum, which was confirmed in 9 patients $(82 \%)$. Fourteen patients had atrophy predicted in the corpus at endoscopy, but only 10 were confirmed by histology (71\%). Figure 2 summarizes the results.

According to MAPS criteria, 12 patients had an extensive disease with premalignant conditions both in antrum and corpus. The concordance of endoscopic classification and histology was $93 \%$ in antrum and $88 \%$ in corpus.

Positive predictive value (PPV) and negative predictive value (NPV) were calculated for each, IM and atrophy. For IM, the PPV and NPV were 0.74 (0.63 in the antrum and 0.875 in the corpus) and 0.83 (0.92 in the antrum and 0.75 in the corpus), respectively, and for atrophy PPV and NPV were 0.63 (0.5 in the antrum and 0.7 in the corpus) and 0.97 (0.94 in the antrum and 1.0 in the corpus), respectively. When disconcordance between histology and endoscopic prediction was present the interval for surveillance according to MAPS guidelines would have been correctly recommended solely based on the endoscopic assessment in all but 1 case. The recommendation in the case described above would have led to an overestimate and therefore to earlier endoscopic follow-up.
One patient demonstrated early-stage gastric cancer, which was diagnosed correctly and 1 patient had a small neuroendocrine tumor that was also identified at endoscopy.

We were able to identify IM and atrophy using the same features previously defined for the use in NBI, for example, light blue crest sign for IM. No additional mucosal features were been identified in our series.

\section{Discussion}

Our preliminary report demonstrates the feasibility of BLI and LCI in the characterization of CG and gastric preneoplastic conditions. However, the results do not suggest that histologic sampling should be omitted but rather that the role of this technique could be to promote more thorough assessment of the gastric mucosa and assist in the interpretation of the histology through careful endoscopic assessment. The reasons why this technique improves on histology alone are manifold. First, histologic assessment of CG and especially the grading of atrophy has very low intraobserver agreement among pathologists, which complicates the comparison between endoscopy and the histologic gold standard [10]. In a study evaluating a special training to improve atrophy grading by pathologists, the kappa value at baseline was
Weigt/Malfertheiner/Canbay/Haybaeck/ Bird-Lieberman/Link 
only 0.19 . After the training, the value increased to 0.43 , which is still not an entirely satisfactory gold standard [10]. This difficulty leads to the second point, which is the lack of a uniform endoscopic classification system for CG in antrum and corpus. Particularly in the gastric corpus, atrophy may be misinterpreted as IM because of the tortuous appearance of glandular structures that appear if early, foveolar atrophy is present. This is reflected in our results, which were best in atrophy prediction in antrum and in prediction of IM in the corpus.

However, we found better results in NPV than PPV, meaning the likelihood of having no IM or atrophy is satisfyingly high if endoscopy does not predict their presence. This can be interpreted as the main clinical impact of these new technologies.

In our study, IM showed the same features at BLI, already known from NBI. These features, such as whitening and/or light blue crest sign, are therefore attributed to the physical properties of the mucosa and are not specific to the imaging modality.

In 2016, Sun et al. [8] published a first attempt of endoscopic classification of distal gastric diseases based on color changes in LCI. Based on the everyday endoscopic practice, we question the usefulness for this simple classification system to fully overcome the difficulties in correct prediction of histology, especially in the antrum because of the problems described above.

Future studies will need to compare the white light endoscopy to LCI and BLI to figure out if these new chromoendoscopy techniques are superior to white light endoscopy.

Some authors noted differences in the detection of lesions in the stomach in white light imaging between light sources using Xenon lamps or multi LED [11]. This can be explained by the different spectrograms of the different types of white lights. While the white light of a Xenon lamp has a homogeneous distribution of light intensity throughout the visible wavelength, the white light that is composed of 4 color LED already emphasizes different wavelength such as 410 and $450 \mathrm{~nm}$. This may already lead to a stronger contrast of vascular structures while still appearing as white light by means of the color spectrum of the image.

From large-scale studies using NBI in CG, it is already known that white light endoscopy is inferior to NBI. However, NBI has certain limitations in identifying lesions for later characterization because of its darker images. LCI may help to detect lesions that can later be characterized with BLI and magnification. Indeed, this has already been proven in studies that investigated LCI and
BLI for the detection and characterization of colon polyps that showed a higher yield of adenoma detection using LCI compared to white light endoscopy [4, 12-14].

Our study, however, is limited by its highly selective nature and its small sample size. Therefore, further prospective studies will be needed to address this question.

In summary, by using BLI and LCI as new imaging modalities, we come closer to our goal of characterizing CG and gastric preneoplastic lesions by integrating endoscopy and histology. Endoscopy plays an important role in identifying lesions and their local distribution, while histology, in most cases, is able to define the detected changes. Endoscopic information is of high importance, as histology alone is not able to assess CG in its full extent. Therefore, an interdisciplinary diagnostic approach combining histological and endoscopic assessment is highly recommended.

\section{Acknowledgement}

The research was supported by the National Institute for Health Research (NIHR) Oxford Biomedical Research Centre (BRC). The views expressed are those of the author(s) and not necessarily those of the NHS, the NIHR or the Department of Health.

\section{Statement of Ethics}

The study adheres to the declaration of Helsinki.

\section{Disclosure Statement}

J.W.: Lecturing fees, scientific grant, and endoscopes from Fujifilm Europe. P.M.: Endoscopic material from Fujifilm. All other authors have no Conflict of interest related to this manuscript.

References
1 Uedo N, Ishihara R, Iishi H, Yamamoto S, Yamamoto S, Yamada T, et al. A new method of diagnosing gastric intestinal metaplasia: narrow-band imaging with magnifying endoscopy. Endoscopy. 2006 Aug;38(8):819-24.

2 Uedo N, Yao K. Endoluminal Diagnosis of Early Gastric Cancer and Its Precursors: Bridging the Gap Between Endoscopy and Pathology. Adv Exp Med Biol. 2016;908:293316

3 Savarino E, Corbo M, Dulbecco P, Gemignani L, Giambruno E, Mastracci L, et al. Narrowband imaging with magnifying endoscopy is accurate for detecting gastric intestinal metaplasia. World J Gastroenterol. 2013 May; 19(17):2668-75. 
4 Yoshida N, Yagi N, Inada Y, Kugai M, Okayama T, Kamada K, et al. Ability of a novel blue laser imaging system for the diagnosis of colorectal polyps. Dig Endosc. 2014 Mar; 26(2):250-8.

5 Yoshida N, Hisabe T, Inada Y, Kugai M, Yagi $\mathrm{N}$, Hirai $\mathrm{F}$, et al. The ability of a novel blue laser imaging system for the diagnosis of invasion depth of colorectal neoplasms. J Gastroenterol. 2014 Jan;49(1):73-80.

6 Bisschops R, Hassan C, Bhandari P, Coron E, Neumann H, Pech O, et al. BASIC (BLI Adenoma Serrated International Classification) classification for colorectal polyp characterization with blue light imaging. Endoscopy. 2018 Mar;50(3):211-20.

7 Ono S, Abiko S, Kato M. Linked color imaging enhances gastric cancer in gastric intestinal metaplasia. Dig Endosc. 2017 Mar;29(2): 230-1.

8 Sun X, Dong T, Bi Y, Min M, Shen W, Xu Y, et al. Linked color imaging application for im- proving the endoscopic diagnosis accuracy: a pilot study. Sci Rep. 2016 Sep;6(1):33473.

9 Dinis-Ribeiro M, Areia M, de Vries AC, Marcos-Pinto R, Monteiro-Soares M, O'Connor A, et al.; European Society of Gastrointestinal Endoscopy; European Helicobacter Study Group; European Society of Pathology; Sociedade Portuguesa de Endoscopia Digestiva. Management of precancerous conditions and lesions in the stomach (MAPS): guideline from the European Society of Gastrointestinal Endoscopy (ESGE), European Helicobacter Study Group (EHSG), European Society of Pathology (ESP), and the Sociedade Portuguesa de Endoscopia Digestiva (SPED). Endoscopy. 2012 Jan;44(1):74-94.

10 Kim SS, Kook MC, Shin OR, Kim HS, Bae HI, Seo A, et al. Factors to improve the interobserver agreement for gastric atrophy and intestinal metaplasia: consensus of definition and criteria. Histopathology. 2018 Apr;72(5): $838-45$.
11 Fukuda H, Miura Y, Hayashi Y, Takezawa T, Ino $\mathrm{Y}$, Okada $\mathrm{M}$, et al. Linked color imaging technology facilitates early detection of flat gastric cancers. Clin J Gastroenterol. 2015 Dec;8(6):385-9.

12 Yoshida N, Naito Y, Yasuda R, Murakami T, Hirose R, Ogiso K, et al. Linked color imaging improves the visibility of various featured colorectal polyps in an endoscopist's visibility and color difference value. Int I Colorectal Dis. 2017 Sep;32(9):1253-60.

13 Suzuki T, Hara T, Kitagawa Y, Takashiro H, Nankinzan R, Sugita O, et al. Linked-color imaging improves endoscopic visibility of colorectal nongranular flat lesions. Gastrointest Endosc. 2017 Oct;86(4):692-7.

14 Min M, Deng P, Zhang W, Sun X, Liu Y, Nong B. Comparison of linked color imaging and white-light colonoscopy for detection of colorectal polyps: a multicenter, randomized, crossover trial. Gastrointest Endosc. 2017 Oct;86(4):724-30. 